\title{
Text and data mining scientific articles with allofplos
}

\author{
Elizabeth Seiver*, M Pacer ${ }^{\S}$, Sebastian Bassi ${ }^{\ddagger}$
}

\begin{abstract}
Mining scientific articles is hard when many of them are inaccessible behind paywalls. The Public Library of Science (PLOS) is a non-profit Open Access science publisher of the single largest journal (PLOS ONE), whose articles are all freely available to read and re-use. allofplos is a Python package for maintaining a constantly growing collection of PLOS's $230,000+$ articles. It also efficiently parses these article files into Python data structures. This article will cover how allofplos keeps your articles up-to-date, and how to use it to easily access common article metadata and fuel your meta-research, with actual use cases from inside PLOS
\end{abstract}

Index Terms-Text and data mining, metascience, open access, science publishing, scientific articles, XML

\section{Introduction}

\section{Why mine scientific articles?}

Scientific articles are the standard mechanism of communication in science. They embody a clear way by which human minds across centuries and continents are able to communicate with one another, growing the total sum of knowledge. Scientific articles are unique resources, in that they are the material artifacts by which this cultural exchange is made concrete and persistent. They offer a unique source of insight into the history of carefully argued, hardwon knowledge. Accordingly because they are made of annotated text, they offer unique opportunities for well-defined text and data mining problems. Importantly, because PLOS represents the largest single journal in the history of publishing, it has collected an excellent corpus for this study, spanning seven journals that specialize in biology and medicine. Equally importantly, because PLOS is Open Access, the opportunity to use this data set is available to anyone capable of downloading and analyzing it. The allofplos library enables more people to do that more easily.

\section{What is allofplos?}

allofplos is a Python package for downloading and maintaining up-to-date scientific article corpora, as well as parsing PLOS XML articles in the JATS (Journal Article Tag Suite) [jats] format. It is available on PyPI [allofplospypi] as well as a GitHub repository [allofplosgh]. Many existing Python packages for parsing XML and/or JATS focus on defensive parsing, where the structure is assumed not to be reliable or the document is immediately

* Corresponding author: elizabeth.seiver@gmail.com

$\S$ Netflix

† Globant

Copyright $@ 2018$ Elizabeth Seiver et al. This is an open-access article distributed under the terms of the Creative Commons Attribution License, which permits unrestricted use, distribution, and reproduction in any medium, provided the original author and source are credited. converted to another intermediate format (often JSON) and XML is just a temporary stepping stone. allofplos uses lxml [lxml05], which is compiled in $\mathrm{C}$, for fast XML parsing and conversion to familiar Python data structures like lists, dictionaries, and datetime objects. The intended audience is researchers who are familiar with scientific articles and Python, but may not be familiar with JATS XML. Other related tools include a parser from fellow Open Access publisher eLife [elife] as well as the Open Access subset for downloading $\mathrm{OA}$ articles in bulk from PubMed Commons (PMC) [pmc].

\section{Functionality}

The primary function of allofplos is to download and maintain a corpus of PLOS articles. To enable users to parse articles without downloading 230,000 XML files, allofplos ships with a starter directory of 122 articles (starterdir), and includes commands for downloading a 10,000 article demo corpus as well. The default path to a corpus is stored as the variable corpusdir in the Python program, and first checks for the environment variable \$PLOS_CORPUS which overrides that default location. If you have used pip to install the program, specifying \$PLOS_CORPUS will ensure that the article data will not be overwritten when you update the allofplos package, as the default location is within the package. (Forking/cloning the GitHub repository avoids this problem, because the default corpus location is in the - gitignore file.)

\section{import os}

os.environ['PLOS_CORPUS'] = 'path/to/corpus_directory' from allofplos import update

update.main ()

Downloading new articles can also be accessed via the command line:

\$ export PLOS_CORPUS="path/to/corpus_directory"

\$ python -m allofplos.update

If no articles are found at the specified corpus location, it will initiate a download of the full corpus. This is a 4.6 GB zip file stored on Google Drive, updated daily via an internal PLOS server, that then is unzipped in that location to around $25 \mathrm{~GB}$ of $230,000+$ XML articles. For incremental updates of the corpus, allofplos first scans the corpus directory for all DOIs (Digital Object Identifiers) [doi] of all articles (constructed from filenames) and compares that with every article DOI from the PLOS search API. The missing articles are then downloaded individually in a rate-limited fashion from links that are constructed using the DOIs. Those files are identical to the ones in the .zip file. The .zip file prevents users from needing to scrape the entire PLOS website for the XML files, and "smartly" scrapes only the latest articles. For a subset of 
provisional articles called "uncorrected proofs", it checks whether the final version is available, and downloads the updated version if so. The files are then ready for parsing and analysis.

\section{Article corpora and parsing}

To initialize a corpus (defaults to corpusdir, or the location set by the \$PLOS_CORPUS environmental variable), use the Corpus class. This points allofplos at the directory of articles to be analyzed.

from allofplos import Corpus

corpus $=$ Corpus ()

To analyze the starter directory, also import starterdir and set corpus $=$ Corpus (starterdir). The number of articles in the corpus can be found with len (corpus). The list of every DOI for every article in the corpus can be found at corpus.dois, and the path to every XML file in the corpus directory at corpus.filenames. To select a random Article object, use corpus.random_article. To select a random list of ten Article objects, use corpus.random_sample (10). You can also iterate through articles as such:

for article in corpus [:10]: print (article.title)

Because DOIs contain semantic meaning and XML filenames are based on the DOI, if you systematically loop through the corpus, it will not be a representative sample but rather will implicitly progress first by journal name and then by publication date. The iterator for Corpus ( ) puts the articles in a random order to avoid this problem.

\section{The Article class}

As mentioned above, you can use the Corpus class to initialize an Article() object without calling Article directly. An Article takes a DOI and the location of the corpus directory to read the accompanying XML document into $\mathrm{lxml}$.

art = Article('10.1371/journal.pcbi.1004692')

The lxml tree of the article is memoized in art.tree so it can be repeatedly called without needing to re-read the XML file.

$>>$ type (art.tree)

lxml.etree._ElementTree

Article parsing in allofplos focuses on metadata (e.g., article title, author names and institutions, date of publication, Creative Commons copyright license [cc], JATS version/DTD), which are conveniently located in the front section of the XML. We designed the parsing API to quickly locate and parse XML elements as properties without needing to know the JATS tagging format.

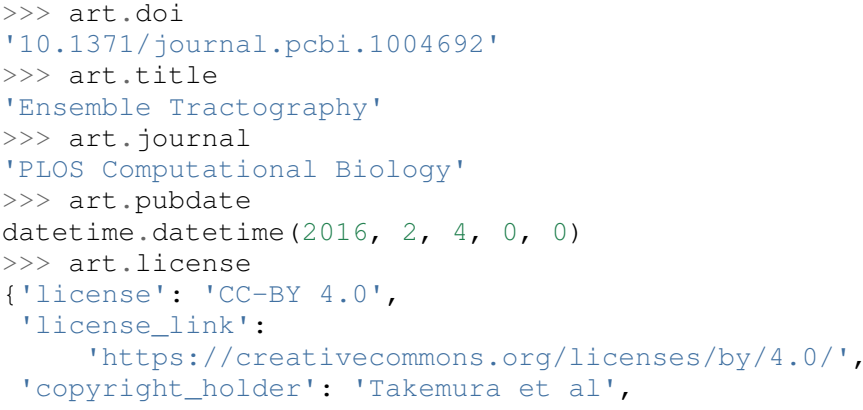

'copyright_year': 2016 \}

$>>$ art.dtd

' JATS 1.1d3'

For author information, Article reconciles and combines data from multiple elements within the article into a clean standard form, including author email addresses and affiliated institutions. Property names match XML tags whenever possible.

\section{Using XPath}

While the Article class handles most basic metadata within the XML files, users may also wish to analyze the content of the article more directly. The XPath query language is built into lxml and provides a way to search for particular XML tags or attributes. (Note that XPath will always return a list of results, as element tags and locations are not unique.) You can perform XPath searches on art.tree, which also works well for finding article elements that are not Article class properties, such as the acknowledgments, which have the tag $\langle a c k\rangle$.

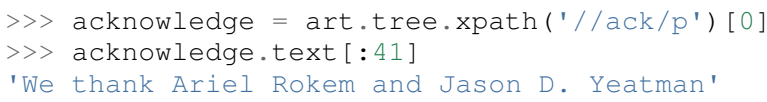

For users who are more familiar with XML or want to perform quality control checks on XML files, XPath searches can find articles that match a particular XML structure. For example, PLOS's production team needed to find articles that had a <list> item anywhere within a $<$ boxed-text $>$ element. They iterated through the corpus using art.tree.xpath ('//boxed-text//list').

\section{Use case: searching Methods sections}

We can put these pieces together to make a list of articles that use PCR (Polymerase Chain Reaction, a common molecular biology technique) in their Methods section (pcr_list). The body of an article is divided into sections (with the element tag $\langle$ sec $\rangle$ ) and the element attributes of Methods sections are either \{'sec-type': 'materials|methods'\} or $\{$ 'sec-type': 'methods'\}. In addition to importing allofplos, the $1 \mathrm{xml}$. etree module needs to be imported to turn XML elements into Python strings via the tostring () method.

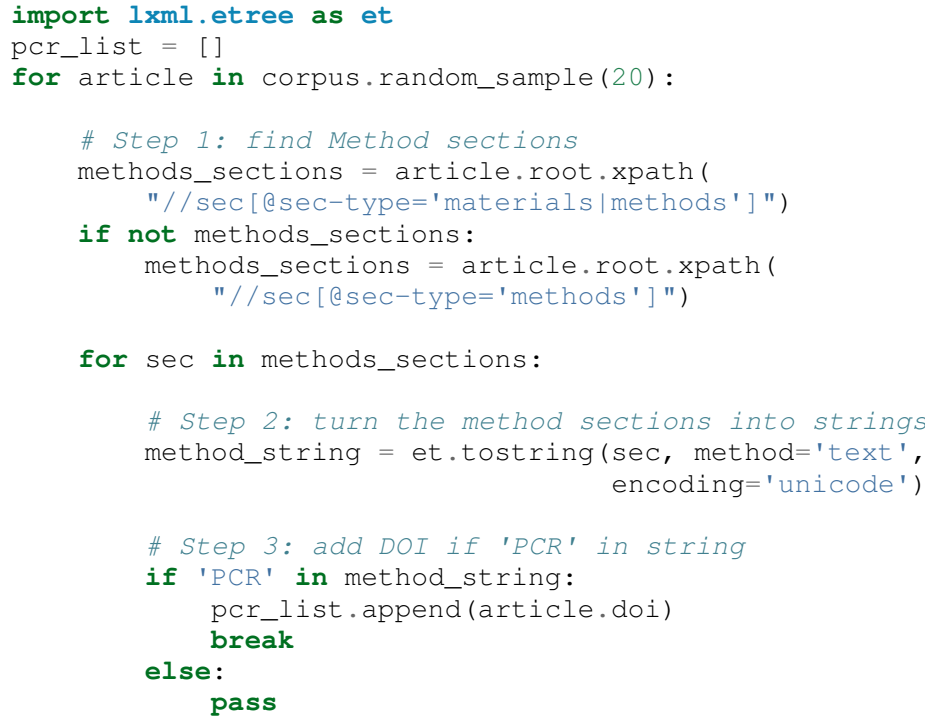




\section{Included SQLite database}

The allofplos code includes a SQLite database with all articles in starter directory. In this release there are 122 records that represents a wide range of papers. In order to use the database, the user needs a SQLite client. The official client is command line based and can be downloaded from https://www.sqlite.org/ download.html. The database can also be displayed on graphical viewers such as DB Browser for SQLite and SQLiteStudio. There are also some options to query the database online, without installing any software, like https://sqliteonline.com/ and http://inloop.github.io/sqlite-viewer/.

The main table of the database is plosarticle. It has the DOI, title, abstract, publication date and other fields that link to other child tables, like articletype and journal_id. The corresponding author information is stored in the correspondingauthor table and is linked to the plosarticle table using the relation table called coauthorplosarticle.

For example, to get all papers whose corresponding authors are from France:

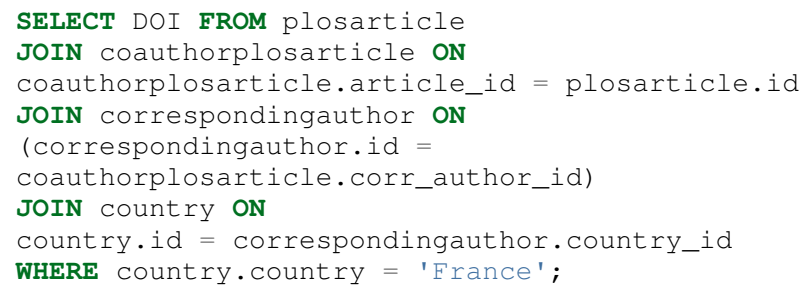

This will return the DOIs from three papers from the starter database:

$10.1371 /$ journal.pcbi.1004152

10.1371 / journal.ppat.1000105

10.1371 / journal.pgen.1002912

10.1371 / journal.pcbi.1004082

The researcher can avoid using SQL queries by using the included Object-relational mapping (ORM) models. The ORM library used is peewee. A file with sample queries is stored in the repository with the name of allofplos/dbtoorm.py. Part of this file defines all Python classes that corresponds to the SQLite Database. These class definitions are from the beginning of the file until the comment marked as \# End of ORM classes creation.

After this comment, there is an example of how to build a query. The following query is the peewee compatible syntax that constructs the same SQL query as outlined before:

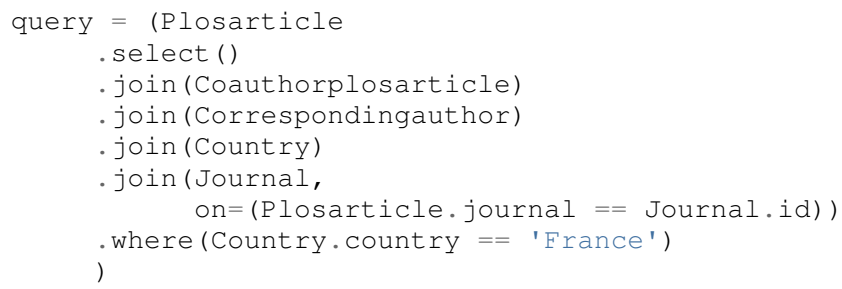

This will return a query object. This object can be walked over with a for loop as any Python iterable:

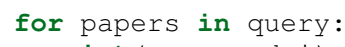

\section{SQLite database constructor}

There is a script at allofplos/makedb.py that can be used to generate the SQLite Database from a directory full of XML articles. This script was used to generate the included starter.db. If the user wants to make another version, from another subset (or from the whole corpus), this script will be useful.

To generate a SQLite DB with all the files currently in the Corpus directory, and save the DB as mydb.db:

\$ python makedb.py --db mydb.db

There is an option to generate a DB with only a random subset of articles. For a DB with 500 articles randomly selected, use:

\$ python makedb.py --random $500--d b$ mydb. db

\section{Future directions}

We also have plans for future updates to allofplos. First, we plan to make the article parsing publisher-neutral, allowing for reading JATS content from other publishers in addition to PLOS. Second, we want to improve incremental corpus updates so that all changes can be downloaded and updated via a standardized mechanism such as a hash table. This includes 'silent republications', where articles are updated online without an official correction notice (the substance of the article is unchanged, but the XML has been updated). While the local allofplos server has methods for catching these changes and updating the zip file appropriately, there is not currently a way to make sure a user's local corpus copy reflects all of those changes. Third, we want to expand the possibilities of multiple corpora and allow for article versioning, such as for comparing older and newer versions of articles instead of just replacing them entirely. And finally, we want to expand and integrate the functionality of the sqlite database so that selecting a subset of articles based on metadata criteria such as journal, publication date, or author is faster and easier than looping through each XML file individually.

\section{Conclusions}

As more scientific articles are published, it will become more important that these articles can be analyzed in aggregate. Tools like allofplos make such an effort much easier. With an intuitive and straightforward Corpus () and Article() APIs, allofplos avoids much of the complexity of parsing $\mathrm{xml}$ for new users, while still enabling XML experts the flexibility and power needed to accomplish their aims. By building in the ability to automatically update and maintain the corpus, people can trust that they have the most state-of-the-art data without needing to manually check the $>230,000$ articles (a task few would undertake). By connecting this information to database technologies, allofplos enables quickly accessing data when that efficient access is needed. By making strides in all of these directions allofplos demonstrates itself to be a valuable tool in the scientific python toolkit.

\section{References}

[1xm105] Behnel, S., Faassen, M. et al. (2005), 1xml: XML and HTML with Python, http://lxml.de.

[cc] Creative Commons Licenses. https://creativecommons.org/ licenses/

[allofplosgh] allofplos GitHub repository. https://github.com/PLOS/ allofplos

[allofplospypi] allofplos PyPI repository. https://pypi.org/project/allofplos/

[jats] JATS NIH/NISO standard. https://jats.nlm.nih.gov/publishing/ tag-library/1.1d3/chapter/how-to-read.html

[elife] elife-tools GitHub repository. https://github.com/elifesciences/ elife-tools 
Digital Object Identifiers. https://www.doi.org/doi_handbook/ 1 Introduction.html

[pmc] PMC Open Access Subset. https://www.ncbi.nlm.nih.gov/ pmc/tools/openftlist/ 